\title{
ẢNH HƯỞNG CỦA CÁC YẾU TỐ CẤU THÀNH VĂN HÓA TỔ CHỨC ĐẾN CHIA SẺ TRI THỨC CỦA GIẢNG VIÊN TRONG TRƯỜNG ĐẠI HỌC: NGHIÊN CÚ̉U TẠI TRƯỜNG ĐẠI HỌC TÀI CHÍNH - MARKETING KEY DETERMINANTS OF ORGANIZATIONAL CULTURE ON THE KNOWLEDGE SHARING OF LECTURERS IN ACADEMIC INSTITUTIONS: A STUDY IN THE UNIVERSITY OF FINANCE AND MARKETING
}

Ngày nhận bài: 11/12/2018 Ngày chấp nhận đăng: 27/12/2018 Ngày đăng: 05/8/2019

Trần Thị Lan Nhung, Nguyễn Thị Cẩm Loan ${ }^{1}$

\section{Tóm tắt}

Nghiên cứu này nhằm xác định các yếu tố của văn hóa tổ chức ảnh hưởng đến chia sẻ tri thức của giảng viên - kiểm định tại trường Đại học Tài chính - Marketing, bằng phương pháp nghiên cứu định tính kết hợp nghiên cứu định lượng. Dữ liệu khảo sát được thu thập từ 215 giảng viên cơ hữu của Trường (trong số hơn 270 giảng viên trước thời điểm Trường Cao đẳng Tài chính - Hải quan sáp nhập vào). Theo đó, các yếu tố cấu thành văn hóa tổ chức ảnh hưởng đến chia sẻ tri thức của giảng viên theo trình tự mức độ ảnh hưởng từ cao xuống thấp là: hệ thống khen thưởng; sự tin tưởng; lãnh đạo và hệ thống thông tin. Bài viết là cơ sở khoa học cho việc kiện toàn văn hóa tổ chức để thúc đẩy chia sẻ tri thức của giảng viên tại trường đại học trong giai đoạn hiện nay.

Tù khóa: Văn hóa tổ chức, chia sẻ tri thức.

\begin{abstract}
This study identifies the critical elements of organizational culture affecting the knowledge sharing between lecturers - the case of the University of Finance - Marketing, conducted by qualitative research combining quantitative research. Survey data was collected from 215 lecturers of the University (out of 270). Accordingly, determinants of elements of organizational culture to the knowledge sharing of the lectures arranged in the order of the level of influence from high to low are: the system of reward; trust; leadership and information systems. The study is a scientific basis for strengthening organizational culture to promote the knowledge sharing at the university in the current period.
\end{abstract}

Key words: Organizational culture, knowledge sharing.

\footnotetext{
${ }^{1}$ Trường ĐH Tài chính - Marketing
} 


\section{Giới thiệu}

Tri thức đóng vai trò thiết yếu trong các tổ chức ngày nay và nó giúp cho các nhà quản lý đưa ra quyết định cần thiết, do đó tri thức là tài sản quý giá nhất và là nền tảng của lợi thế cạnh tranh của một tổ chức (Bock và cộng sự, 2005). Tuy nhiên, con người không sẵn sàng chia sẻ những tri thức mà họ đã tích lũy được bởi tính cá nhân và tính sở hữu luôn tồn tại trong mỗi con người. Họ sợ rằng sẽ mất đi sức mạnh tri thức của họ trong tổ chức nếu chia sẻ với người khác (Davenport và công sự, 1998). Dẫn đến, chia sẻ tri thức được xem là một trong những hoạt động khó khăn nhất (Ruggles,1998) mặc dù chia sẻ tri thức được coi là yếu tố quan trọng, tạo điều kiện phát huy tối đa khả năng sáng tạo, trí tuệ và kinh nghiệm của mọi thành viên trong tổ chức.

Trường đại học, là những tổ chức tích hợp cho việc nghiên cứu, học tập và học hỏi về tài sản kiến thức. Chia sẻ tri thức là vấn đề quan trọng trong mọi tổ chức, đặc biệt là trong môi trường giáo dục đại học. Trên cơ sở tổng kết từ nhiều nghiên cứu khác nhau đã chứng minh rằng văn hóa tổ chức có vai trò rất quan trọng, tạo ra môi trường làm việc thúc đẩy chia sẻ tri thức giữa các nhân viên trong tổ chức. Mối quan hệ giữa văn hóa tổ chức và chia sẻ tri thức đã được nghiên cứu nhiều trên thế giới. Tuy nhiên, cho đến hiện nay, ở trong nước, dường như vẫn chưa có những nghiên cứu chính thức về vấn đề đề này trong lĩnh vực giáo dục đại học. Do đó, rất cần có một nghiên cứu chính thức về mối liên hệ này cho trường hợp trường đại học tại Việt Nam. Kết quả nghiên cứu này là cơ sở khoa học cho việc kiện toàn văn hóa tổ chức để thúc đẩy chia sẻ tri thức của giảng viên tại trường đại học trong giai đoạn hiện nay.

\section{Cơ sở lý thuyết và mô hình nghiên cứu}

Văn hóa tổ chức đã trở thành một khái niệm trong khoa học tổ chức - quản lý xuất hiện ở Âu Mỹ từ những năm 80 của thế kỷ trước và hiện nay là một khái niệm thịnh hành và được phổ biến rộng rãi. Văn hóa tổ chức có rất nhiều khái niệm khác nhau tùy theo cách tiếp cận. Nhưng tựu trung lại từ quan điểm của nhiều nhà nghiên cứu văn hóa tổ chức là hệ thống niềm tin, giá trị, chuẩn mực, thói quen và truyền thống được tạo ra trong quá trình lịch sử, được các thành viên trong nhà trường thừa nhận, làm theo và in dấu ấn trong các hình thái vật chất và tinh thần (Eldrige và Crombie,1974), (Schein,1984), (Luthans, 1992), (Ricardo và Jolly, 1997), (Lawson và Shen, 1998).

Chia sẻ tri thức là một trong những hoạt động chính, quan trọng của quản lý tri thức (Alavi và Leidner, 2006; Becerra-Fernandez và cộng sự, 2004; Lee và cộng sự, 2002; Gupta và cộng sự, 2000). Chia sẻ tri thức là hành động chủ quan cố ý làm cho tri thức được tái sử dụng bởi những người khác thông qua chuyển giao tri thức là quá trình cho và nhận tri thức, trong đó sự sáng tạo và chia sẻ tri thức phụ thuộc vào nỗ lực có ý thức của cá nhân làm cho tri thức được chia sẻ.

Nghiên cứu của Al-Alawi và cộng sự (2007) "Văn hóa tổ chức và chia sẻ tri thức: các yếu tố quyết định thành công" đã dựa vào mô hình của Gupta và Govindarajan (2000), đồng thời kế thừa kết quả các nghiên cứu trước để khám phá các yếu tố ảnh hưởng của văn hóa tổ chức đến hành vi chia sẻ tri thức của nhân viên làm việc trong các tổ chức thuộc khu vực công và các doanh nghiệp thuộc khu vực tư ở Vương quốc Bahrain. Kết quả nghiên cứu chỉ ra rằng các yếu tố văn hóa tổ chức: sự tin tưởng, giao tiếp, cấu trúc tổ chức, hệ thống khen thưởng đều có tác 
động tích cực với việc chia sẻ tri thức trong tổ chức, còn yếu tố hệ thống thông tin có tác động chưa rõ ràng đến chia sẻ tri thức.

Nghiên cứu của Islam và cộng (2011) "Văn hóa tổ chức và chia sẻ tri thức: bằng chứng thực nghiệm tù các tổ chức dịch vụ" được thực hiện tại 7 tổ chức dịch vụ ở Bangladesh nhằm mục đích kiểm tra mối quan hệ giữa các yếu tố văn hóa tổ chức và chia sẻ tri thức. Kế thừa kết quả nghiên cứu của Al-Alawi và cộng sự (2007) tác giả này đã xây dựng mô hình nghiên cứu sự tác động của 4 yếu tố văn hóa tổ chức đến chia sẻ tri thức: sự tin tưởng, giao tiếp giữa các nhân viên, lãnh đạo, hệ thống khen thưởng. Kết quả nghiên cứu cho thấy các yếu tố có tác động thúc đẩy chia sẻ tri thức là sự tin tưởng, giao tiếp và lãnh đạo. Trong khi các nghiên cứu trước đều cho thấy hệ thống khen thưởng có tác động tích cực đến chia sẻ tri thức thì trong nghiên cứu này hệ thống khen thưởng lại tác động không có ý nghĩa đến chia sẻ tri thức.

Nghiên cứu của Kathiravelu và cộng sự (2014) "Tại sao văn hóa tổ chức có thể dẫn đến chia sẻ tri thức?". Đối tượng khảo sát là nhân viên và các thành viên dịch vụ công cộng, chủ yếu là các dịch vụ dân sự tại Malaysia. Các yếu tố văn hóa tổ chức bao gồm: sự tin tưởng, giao tiếp, lãnh đạo, cấu trúc tổ chức, hệ thống khen thưởng và hệ thống thông tin. Kết quả nghiên cứu cho thấy có một mối quan hệ chặt chẽ giữa văn hóa tổ chức và chia sẻ tri thức của các thành viên trong tổ chức.

Nghiên cứu của Trần Minh Thành (2013) "Anh huơong của các yếu tố văn hóa tổ chưc đến chia sẻ tri thức - Nghiên cứu tại các doanh nghiệp xây dựng TP. Hồ Chí Minh" cho thấy có 5 yếu tố của văn hóa tổ chức có tác động tích cực đến chia sẻ tri thức đó là: khen thưởng tinh thần có tác động mạnh nhất, thứ hai là sự tin tưởng, tiếp theo là lãnh đạo, quy trình làm việc và cuối cùng là giao tiếp. Còn yếu tố khen thưởng vật chất được kết luận là tác động không có ý nghĩa đến chia sẻ tri thức

Từ cơ sở lý thuyết và tổng quan các nghiên cứu trước, kết hợp với các đặc điểm của nghề nghiệp giảng viên dưới góc độ văn hóa tổ chức và chia sẻ tri thức, tác giả đề xuất mô hình các yếu tố cấu thành văn hóa tổ chức ảnh hưởng đến hành vi chia sẻ tri thức của giảng viên trong trường đại học kế thừa từ mô hình nghiên cứu của Kathiravelu và cộng sự (2014). Theo đó, văn hóa tổ chức trong trường đại học bao gồm 6 yếu tố: sự tin tưởng, hệ thống thông tin, hệ thống khen thưởng, lãnh đạo, giao tiếp và cấu trúc tổ chức. Trong đó:

\subsection{Sỵ tin tưởng}

Sự tin tưởng và hợp tác dẫn đến một sự sẵn sàng lớn hơn cho các nhân viên để chia sẻ những hiểu biết và kinh nghiệm với nhau (Delong và Fahey, 2000). Thang đo sự tin tưởng gồm 06 biến quan sát từ TTG1-TTG6 được phát triển từ thang đo của Al-adaileh (2011).

TTG1: Tôi không ngần ngại chia sẻ cảm xúc và nhận thức của tôi với các đồng nghiệp trong trường.

TTG2: Tôi tin rằng việc chia sẻ tri thức sẽ giúp tôi trong sự nghiệp của mình.

TTG3: Tin tưởng lẫn nhau giữa các đồng nghiệp là cần thiết để tri thức được trao đổi tự do trong trường.

TTG4: Tôi tin rằng mỗi đóng góp và nỗ lực chia sẻ những hiểu biết của tôi sẽ được đồng nghiệp đánh giá cao.

TTG5: Lãnh đạo nhà trường tin tưởng vào khả năng chia sẻ tri thức của các giảng viên. 
TTG6: Môi trường làm việc trong nhà trường giúp giảng viên tin tưởng chia sẻ tri thức với nhau.

\section{Giả thuyết H1: Sự tin tưởng có ảnh hưởng} cùng chiều đến chia sẻ tri thức của giảng viên

\subsection{Hệ thống công nghệ thông tin}

Nhờ vào công nghệ thông tin, chia sẻ tri thức có thể diễn ra ở bất cứ đâu và bất cứ khi nào. Davenpork và Prusak (1998) cho rằng hệ thống công nghệ thông tin có mối quan hệ cùng chiều với chia sẻ tri thức, sẽ cải thiện được hiệu suất của tổ chức và làm gia tăng tỷ lệ chia sẻ tri thức trong tổ chức đó. Thang đo hệ thống thông tin gồm 04 biến quan sát từ TTN1-TTN4 được phát triển từ thang đo của Al-Alawi và cộng sự (2007); Lee và Choi (2003).

TTN1: Nhà trường cung cấp đầy đủ các trang thiết bị cần thiết hỗ trợ cho việc chia sẻ tri thức của giảng viên với nhau.

TTN2: Hệ thống công nghệ thông tin của trường cung cấp những thông tin, dữ liệu hữu ích cho việc chia sẻ tri thức của giảng viên.

TTN3: Hệ thống công nghệ thông tin của trường giúp cho việc chia sẻ tri thức giữa các giảng viên với nhau có hiệu quả.

TTN4: Giảng viên chủ yếu sử dụng hệ thống công nghệ thông tin của nhà trường để chia sẻ tri thức với đồng nghiệp.

Giả thuyết H2: Hệ thống thông tin có ảnh hưởng cùng chiều đến chia sẻ tri thức của giảng viên

\subsection{Hệ thống khen thưởng}

Hệ thống khen thưởng là một tập hợp các ưu đãi cần thiết dành cho thành viên trong tổ chức nhằm định hướng cho hành vi của họ hoặc là cải thiện hiệu quả trong học tập (Jahani và cộng sự (2011). Để khuyến khích nhân viên sáng tạo tri thức, chia sẻ tri thức thì tổ chức cần tôn trọng nhân viên, có hình thức khen thưởng kịp thời cho nhân viên khi họ đóng góp những sáng kiến quan trọng cho tổ chức. Thang đo hệ thống khen thưởng gồm 05 biến quan sát từ KTH1KTH5 được phát triển từ thang đo của Al-Alawi và cộng sự (2007), Jahani và cộng sự (2011):

KTH1: Giảng viên được khen thưởng khi họ chia sẻ tri thức của mình với các đồng nghiệp.

KTH2: Nhà trường có các chính sách khuyến khích giảng viên chia sẻ tri thức với nhau.

KTH3: Phần thưởng của Nhà trường giúp tôi tự tin hơn trong việc chia sẻ tri thức với đồng nghiệp.

KTH4: Càng chia sẻ tri thức tôi càng được nhà trường ghi nhận nhiều hơn.

KTH5: Chế độ khen thưởng của Nhà trường kích thích giảng viên chia sẻ tri thức với nhau.

Giả thuyết H3: Hệ thống khen thưởng có ảnh hưởng cùng chiều đến chia sẻ tri thức của giảng viên

\subsection{Lãnh đạo}

Phong cách lãnh đạo phù hợp được xem là một trong những nhân tố quan trọng nhất ảnh hưởng đến hiệu quả quản lý tri thức trong tổ chức. Một số nghiên cứu như của Jahani và cộng sự (2011) cho thấy yếu tố lãnh đạo có vai trò quan trọng trong việc chia sẻ tri thức trong tổ chức. Thang đo lãnh đạo gồm 05 biến quan sát từ LDO1- LDO5 được phát triển từ thang đo của Jahani và cộng sự (2011); Donate và Guadamillas (2011).

LDO1: Lãnh đạo Nhà trường rất cởi mở đối với giảng viên trong việc chia sẻ tri thức. 
LDO2: Lãnh đạo Nhà trường khích lệ, tuyên dương kịp thời đối với các giảng viên có ý tưởng, sáng kiến trong công việc cho đơn vị.

LDO3: Lãnh đạo Nhà trường tôn trọng ý kiến, quan điểm của giảng viên.

LDO4: Lãnh đạo Nhà trường khuyến khích giảng viên hợp tác với nhau trong công việc.

LDO5: Lãnh đạo Nhà trường luôn tạo điều kiện, cơ hội để giảng viên chia sẻ tri thức với nhau.

Giả thuyết H4: Lãnh đạo có ảnh hưởng cùng chiều đến chia sẻ tri thức của giảng viên

\subsection{Giao tiếp}

Greenberg và Baron (2003) cho rằng giao tiếp có tác động đến thái độ của các nhân đối với tổ chức. Giao tiếp giúp tạo ra sự chia sẻ, các quy tắc, các giá trị và văn hóa của tổ chức Wiesenfeld (1999). Thang đo giao tiếp gồm 05 biến quan sát từ GTP1- GTP5 được phát triển từ thang đo của Al-Alawi và cộng sự (2007).

GTP1: Giảng viên có nhiều cơ hội để tương tác mặt đối mặt với đồng nghiệp.

GTP2: Giảng viên được tăng cường khả năng giao tiếp, thúc đẩy chia sẻ tri thức với nhau thông qua các sự kiện khoa học.

GTP3: Khác biệt về tuổi tác, vùng miền, trình độ học vấn, chức vụ không phải là một rào cản trong giao tiếp giữa các giảng viên với nhau.

GTP4: Có nhiều hình thức khác nhau để giảng viên có thể chia sẻ tri thức với nhau.

GTP5: Tôi cảm thấy, các giảng viên đều hợp tác chặt chẽ với nhau trong công việc.
Giả thuyết H5: Giao tiếp có ảnh hưởng cùng chiều đến chia sẻ tri thức của giảng viên

\subsection{Cấu trúc tổ chức}

Cấu trúc tổ chức là một hệ thống chính thức về các mối quan hệ vừa độc lập, vừa phụ thuộc trong tổ chức, thể hiện những nhiệm vụ rõ ràng do ai làm, làm cái gì và liên kết với các nhiệm vụ khác trong tổ chức như thế nào nhằm tạo ra một sự tập hợp nhịp nhàng để đáp ứng mục tiêu của tổ chức. Syed-Ikhsan và cộng sự (2004), Al-Alawi và cộng sự (2007) và Mueller (2012) lập luận rằng cấu trúc tổ chức ảnh hưởng tích cực đến chia sẻ tri thức. Thang đo cấu trúc tổ chức gồm 04 biến quan sát từ TCH1-TCH5 được phát triển từ thang đo của Al-Alawi và cộng sự (2007).

TCH1: Cơ cấu giảng viên theo các đơn vị (khoa, ngành) rất hợp lý cho việc chia sẻ tri thức giữa họ với nhau.

TCH2: Việc chia sẻ tri thức giữa các giảng viên với nhau thực hiện một cách dễ dàng trong Nhà trường bất kể giảng viên là nhân viên hay lãnh đạo.

TCH3: Các giảng viên dễ dàng liên hệ với nhau khi có nhu cầu chia sẻ tri thức.

TCH4: Một số nhiệm vụ của Nhà trường đòi hỏi sự hình thành đội, nhóm trong đó có sự phối hợp giữa các giảng viên ở các khoa, phòng, ban khác nhau.

Giả thuyết H6: Cấu trúc tổ chức có ảnh hưởng cùng chiều đến chia sẻ tri thức của giảng viên 


\subsection{Mô hình lý thuyết tác giả đề xuất}

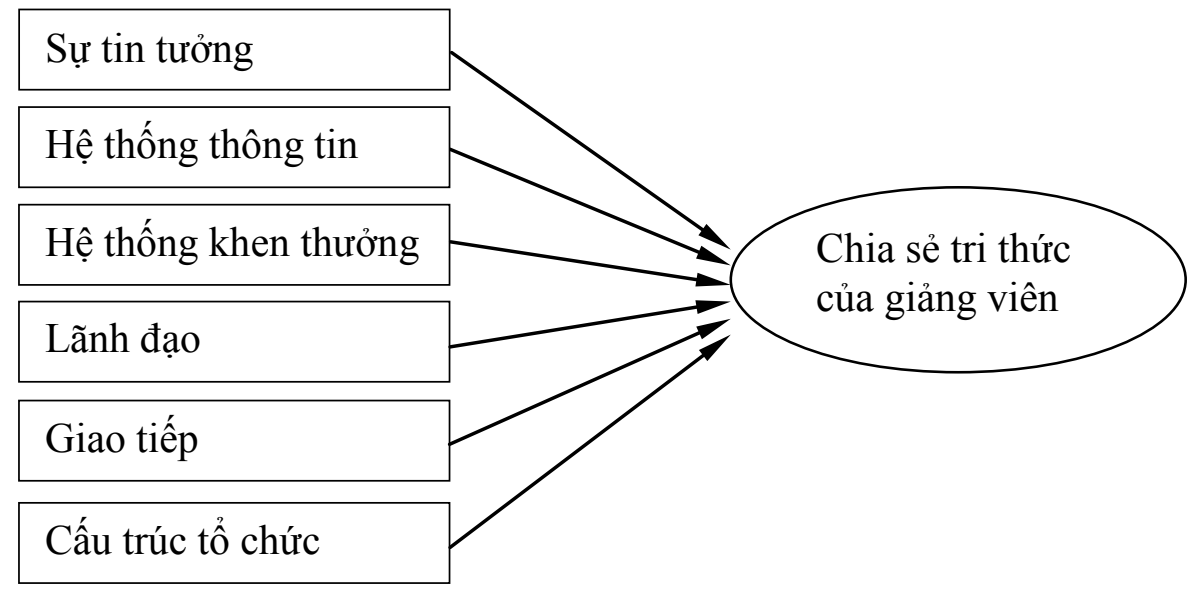

\section{Hình 1. Mô hình lý thuyết các yếu tố ảnh hưởng đến chia sẻ tri thức của giảng viên tại trường Đại học Tài chính - Marketing}

Nguồn: Đề xuất của tác giả

\section{Phương pháp nghiên cứu}

Nghiên cứu sử dụng chủ yếu phương pháp nghiên cứu định tính kết hợp nghiên cứu định lượng.

- Nghiên cứu định tính: sử dụng kỹ thuật thảo luận nhóm tập trung với 02 nhóm (mỗi nhóm gồm 08 giảng viên cơ hữu đang dạy tại Trường Đại học Tài chính - Marketing) nhằm điều chỉnh, bổ sung các yếu tố ảnh hưởng của văn hóa tổ chức đến chia sẻ tri thức của giảng viên tại Trường Đại học Tài chính - Marketing và phát triển thang đo những yếu tố này.

- Phương pháp nghiên cứu định lượng: Để tiến hành phân tích hồi quy, theo Tabachnick và Fidell kích thước mẫu phải bảo đảm theo công thức: $\mathrm{n} \geq 8 \mathrm{~m}+50$ (với $\mathrm{m}$ là số biến độc lập trong mô hình); theo Harris RJ thì $\mathrm{n} \geq 104+\mathrm{m}$ (với $\mathrm{m}$ là số lượng biến độc lập và phụ thuộc). Để phân tích EFA, theo Hair và cộng sự $\mathrm{n} \geq 5 \times \mathrm{X}$ (với $\mathrm{X}$ là tổng số biến quan sát). Trong mô hình nghiên cứu này có 6 biến độc lập, 1 biến phụ thuộc, 35 biến quan sát. Vì thế, theo Tabachnick và Fidell là $\mathrm{n} \geq 98$; theo Harris RJ $\mathrm{n} \geq 111$; theo Hair và cộng sự, $\mathrm{n} \geq 175$. Dữ liệu nghiên cứu được thu thập bằng hình thức phỏng vấn bằng bảng câu hỏi cho 270 giảng viên cơ hữu của Trường (trước khi sáp nhập với Cao đẳng Tài chính Hải quan đầu năm 2018) và nhận về được 215 bản trả lời hợp lệ. Vậy 215 phiếu trả lời đã đáp ứng tốt yêu cầu để phân tích.

Phương pháp nghiên cứu định lượng nhằm đánh giá độ tin cậy thang đo của các yếu tố văn hóa tổ chức đến chia sẻ tri thức; kiểm định mô hình nghiên cứu và các giả thuyết nghiên cứu.

Quá trình phân tích dữ liệu nghiên cứu sử dụng SPSS với các kỹ thuật: Cronbach's alpha và phân tích nhân tố khám phá (EFA Exploratory Factor Analysis) nhằm kiểm định độ tin cậy của các thang đo; phân tích hồi qui bội nhằm kiểm định mô hình nghiên cứu, các giả thuyết nghiên cứu và định vị tầm quan trọng của các yếu tố ảnh hưởng.

\section{Kết quả nghiên cứu}

- Kết quả nghiên cứu định tính khẳng định 6 yếu tố được đề xuất trong mô hình lý thuyết 
(hình 1) đều là các yếu tố văn hóa tổ chức chính ảnh hưởng đến chia sẻ tri thức của giảng viên; đồng thời, phát triển thang đo (thang đo Likert 7 bậc từ $1 \div 7 ; 1$ là hoàn toàn phản đối; 7 là hoàn toàn đồng ý) các yếu tố này (gồm 29 biến quan sát) và biến phụ thuộc là chia sẻ tri thức của giảng viên (gồm 6 biến quan sát).

- Kết quả Cronbach's alpha cho thấy các thang đo đều đạt độ tin cậy (thấp nhất là thang đo Hệ thống thông tin $\alpha=0,834$; cao nhất là thang đo Lãnh đạo $\alpha=0,914$ ).

- Kết quả EFA thang đo các yếu tố văn hóa tổ chức và thang đo chia sẻ tri thức của giảng viên bằng phương pháp trích Principal Axis Factoring và phép quay Promax cho thấy cho thấy:

+ Lần 1: Kết quả EFA lần 1 cho thấy 2 biến TTG4 và KTH4 có hệ số tải nhân tố nhỏ hơn $0,4,4$ biến còn lại GTP1; GTP2; LDO5 và CST3 có hệ số tải nhân tố lớn hơn 0,4 nhưng chênh lệch của biến quan sát giữa các nhân tố nhỏ hơn 0,3 .

+ Lần 2: Sau khi loại các biến TTG4; KTH4; GTP1; GTP2; LDO5 và CST3, kết quả EFA lần 2 cho thấy biến CST5 có hệ số tải nhân tố nhỏ hơn 0,4 .

+ Lần 3: Sau khi loại biến CST5, kết quả phân tích nhân tố EFA lần 3 cho thấy, chỉ số $\mathrm{KMO}=0,905$ với mức ý nghĩa Sig. $=0,000$, đồng thời các tập biến quan sát được rút trích vào 7 nhân tố nguyên gốc tại eigenvalue là 1,003 với tổng phương sai trích được là $67,863 \%$. Điều này cho phép thang đo các yếu tố ảnh hưởng của văn hóa tổ chức và chia sẻ tri thức của giảng viên đạt yêu cầu cho bước phân tích hồi qui tiếp theo.

Như vậy, với kết quả phân tích EFA trên đây thì các yếu tố văn hóa tổ chức ảnh hưởng đến chia sẻ tri thức của giảng viên được giữ nguyên gốc như trong mô hình nghiên cứu đề xuất, nhưng thang đo chúng có hiệu chỉnh. Cụ thể là:

Yếu tố sự tin tưởng gồm 5 biến quan sát: TTG1, TTG2, TTG3, TTG5, TTG6.

Yếu tố hệ thống thông tin gồm 4 biến quan sát: TTN1, TTN2, TTN3, TTN4.

Yếu tố hệ thống khen thưởng gồm 4 biến quan sát: KTH1, KTH2, KTH3, KTH5.

Yếu tố lãnh đạo gồm 4 biến quan sát: LDO1, LDO2, LDO3, LDO4.

Yếu tố giao tiếp gồm 3 biến quan sát: GTP3, GTP4, GTP5.

Yếu tố cấu trúc tổ chức gồm 4 biến quan sát: TCH1, TCH2, TCH3, TCH4.

Yếu tố chia sẻ tri thức gồm 4 biến quan sát: CST1, CST2, CST4, CST6.

- Kết quả kiểm tra hệ số tương quan giữa các yếu tố của văn hóa tổ chức và chia sẻ tri thức của giảng viên có hệ số Pearson ở mức trung bình (dao động từ 0,474 đến 0,537 ) và đều có sig. $=0,000$, chứng các biến độc lập có mối quan hệ khá chặt chẽ với biến phụ thuộc. Đồng thời, hệ số tương quan các biến độc lập với nhau cũng ở mức trung bình (dao động từ 0,302 đến 0,531 ). Vì thế ít có khả năng xảy ra hiện tượng đa cộng tuyến, đồng thời các yếu tố đề xuất trong mô hình lý thuyết có nhiều khả năng giải thích cho việc chia sẻ tri thức.

- Kết quả phân tích hồi qui thu được: $\mathrm{R}^{2}=0,539 ; \mathrm{R}^{2}$ điều chỉnh $=0,526$; giá trị 40,50. Tuy nhiên, trong 6 yếu tố trên có 2 yếu tố là giao tiếp (GTP) và cấu trúc tổ chức $(\mathrm{TCH})$ đều có giá trị sig $>0,05$ rất nhiều nên không có ý nghĩa về mặt thống kê. 
Bảng 1. Kết quả các thông số hồi quy

\begin{tabular}{|c|c|c|c|c|c|c|c|}
\hline \multirow{2}{*}{ Biến } & \multicolumn{2}{|c|}{ Hệ số chưa chuẩn hóa } & $\begin{array}{c}\text { Hệ số } \\
\text { chuẩn hóa }\end{array}$ & \multirow{2}{*}{ t } & \multicolumn{2}{|c|}{ Sig. } & \multicolumn{2}{|c|}{ Đa cộng tuyến } \\
\cline { 2 - 4 } & B & Std. Error & Beta & & & $\begin{array}{c}\text { Độ chấp } \\
\text { nhận }\end{array}$ & \multirow{2}{*}{ VIF } \\
\hline (Constant) & $-0,019$ & 0,337 & & $-0,057$ & 0,955 & & \\
\hline TTG & 0,278 & 0,070 & 0,249 & 3.983 & 0,000 & 0,567 & 1,765 \\
\hline TTN & 0,137 & 0,063 & 0,136 & 2.176 & 0,031 & 0,567 & 1,764 \\
\hline KTH & 0,326 & 0,057 & 0,350 & 5.737 & 0,000 & 0,596 & 1,677 \\
\hline LDO & 0,150 & 0,046 & 0,187 & 3.283 & 0,001 & 0,684 & 1,461 \\
\hline GTP & 0,016 & 0,052 & 0,018 & 0,314 & 0,754 & 0,685 & 1,461 \\
\hline TCH & 0,022 & 0,053 & 0,027 & 0,421 & 0,674 & 0,550 & 1,817 \\
\hline
\end{tabular}

Sau khi loại 2 yếu tố giao tiếp (GTP) và cấu trúc tổ chức (TCH), kết quả hồi quy cho thấy mô hình 4 yếu tố được chọn $\mathrm{R}^{2}$ hiệu chỉnh 0,529 , có giá trị 61,162 tại mức ý nghĩa Sig. < 0,05, nghĩa là mô hình 4 biến độc lập này giải thích được
$52,9 \%$ biến thiên của biến phụ thuộc. Như vậy 2 yếu tố giao tiếp (GTP) và cấu trúc tổ chức (TCH) chỉ giải thích được $0,3 \%$ biến thiên biến phụ thuộc, do đó ta có cơ sở hợp lý để loại 2 yếu tố này ra khỏi mô hình.

Bảng 2. Kết quả các thông số hồi quy

\begin{tabular}{|c|c|c|c|c|c|c|c|}
\hline \multirow{2}{*}{ Biến } & \multicolumn{2}{|c|}{ Hệ số chưa chuẩn hóa } & \multirow{2}{*}{$\begin{array}{c}\begin{array}{c}\text { Hệ số } \\
\text { chuẩn hóa }\end{array} \\
\text { Beta }\end{array}$} & \multirow{2}{*}{$\mathbf{t}$} & \multirow{2}{*}{ Sig. } & \multicolumn{2}{|c|}{ Đa cộng tuyến } \\
\hline & B & Std. Error & & & & $\begin{array}{c}\text { Độ chấp } \\
\text { nhận }\end{array}$ & VIF \\
\hline (Constant) & $-0,017$ & 0,330 & & $-0,051$ & 0,959 & & \\
\hline TTG & 0,298 & 0,060 & 0,267 & 4,975 & 0,000 & 0,765 & 1,308 \\
\hline TTN & 0,142 & 0,062 & 0,141 & 2,283 & 0,023 & 0,578 & 1,729 \\
\hline LDO & 0,153 & 0,045 & 0,191 & 3,384 & 0,001 & 0,694 & 1,442 \\
\hline KTH & 0,334 & 0,054 & 0,358 & 6,137 & 0,000 & 0,645 & 1,551 \\
\hline
\end{tabular}

Nguồn: Kết quả phân tích dũ liệu

Kết quả phân tích hồi qui cũng cho thấy các giá trị $\mathrm{B}$ và Beta đều dương, đồng thời các giá trị kiểm định $\mathrm{t}$ đều có ý nghĩa thống kê. Điều này chứng tỏ các giả thuyết $\mathrm{H} 1, \mathrm{H} 2, \mathrm{H} 3, \mathrm{H} 4$, đều được chấp nhận và phương trình hồi qui dạng chưa chuẩn hóa về các yếu tố ảnh hưởng đến chia sẻ tri thức của giảng viên tại trường Đại học Tài chính - Marketing được xác định như sau:

$$
\mathrm{CST}=0,298 * \mathrm{TTG}+0,142 * \mathrm{TTN}+0,334 * \mathrm{KTH}+0,153 * \mathrm{LDO}
$$




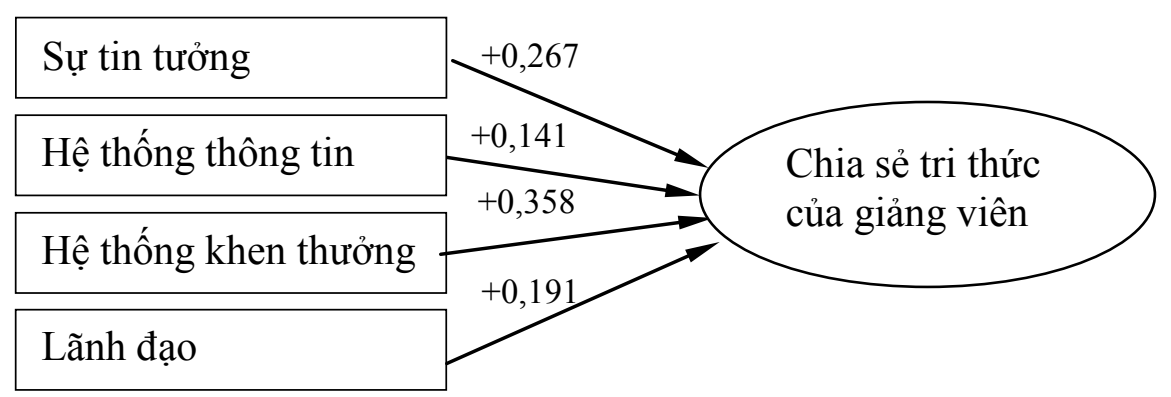

\section{Hình 2. Mô hình nghiên cứu sau phân tích hồi quy}

- Kết quả kiểm tra các vi phạm giả định của mô hình hồi qui cho thấy các giả định này đều không vi phạm. Vì thế, mô hình hồi qui và các giả thuyết nghiên cứu được kiểm định trong nghiên cứu này được chấp nhận.

\section{Kết luận và một số hàm ý}

Kết quả nghiên cứu cho thấy có sự khác biệt với các nghiên cứu trước đây, đặc biệt là đối với nghiên cứu kế thừa Kathiravelu và cộng sự, trong đó 6 yếu tố của văn hóa tổ chức đều có ý nghĩa thống kê thì nghiên cứu của tác giả lại bác bỏ yếu tố giao tiếp và cấu trúc tổ chức.

Từ kết quả phân tích, tác giả xin đề xuất một số hàm ý quản trị nhằm thúc đẩy hoạt động chia sẻ tri thức giữa các giảng viên trong trường Đại học Tài chính - Marketing như sau:

Thư nhất, Nhà trường cần có những chính sách khen thưởng đúng đắn, phù hợp nhằm khích lệ tinh thần chia sẻ tri thức của giảng viên. Hệ thống khen thưởng là yếu tố tác động mạnh nhất đến chia sẻ tri thức với hệ số beta $=0,358$. Tuy nhiên thực tế giảng viên tại trường Đại học Tài chính - Marketing chỉ đánh giá ở mức trung bình Mean $=4,7058$. Điều này cho thấy, giảng viên đánh giá thấp yếu tố khen thưởng trong hoạt động chia sẻ tri thức của họ. Nguyên nhân là do giảng viên chưa được khen thưởng đúng và kịp thời khi họ chia sẻ tri thức với đồng nghiệp; phần thưởng của trường Đại học Tài chính - Marketing cho giảng viên chưa tương xứng với những gì họ cống hiến nên chưa giúp họ tự tin trong việc chia sẻ tri thức; chế độ khen thưởng của Nhà trường cũng chưa kích thích giảng viên chia sẻ tri thức.

Thư hai, các nhà quản lý cần chú trọng phát triển yếu tố sự tin tưởng, xây dựng môi trường làm việc cởi mở, thoải mái, dựa trên sự tin tưởng để hoạt động chia sẻ tri thức diễn ra thường xuyên và ngày một hiệu quả hơn. Sự tin tưởng là yếu tố tác động mạnh đến chia sẻ tri thức với hệ số beta $=0,267$, đồng thời các giảng viên đánh giá yếu tố này ở mức độ trung bình Mean $=5,6660$.

Kết quả phân tích dữ liệu cho thấy, giảng viên đánh giá rất cao sự tin tưởng trong hoạt động chia sẻ tri thức của họ. Vì thế, các nhà quản trị cần chú trọng phát triển yếu tố sự tin tưởng, xây dựng môi trường làm việc cởi mở, thoải mái, dựa trên sự tin tưởng; xây dựng môi trường làm việc tích cực với sự thống nhất rõ ràng, minh bạch trong mọi hoạt động của tổ chức, phân chia chức năng và quyền hạn rõ ràng. Môi trường làm việc đó dựa trên sự tin tưởng, giảng viên tin tưởng lãnh đạo và đồng nghiệp, tin tưởng vào sự tồn tại và phát triển không ngừng của nhà trường. Nhà trường cần xây dựng một nền văn hóa tổ chức phù hợp, 
đề cao sự hợp tác làm việc giữa các giảng viên một cách tự nguyện, thoải mái sẽ thúc đẩy sự trao đổi giữa các giảng viên, gia tăng niềm tin lẫn nhau, là cơ sở để cải thiện hiệu quả chia sẻ tri thức.

Thư $b a$, Nhà trường cần nâng cao vai trò của lãnh đạo trong hoạt động chia sẻ tri thức của giảng viên. Kết quả phân tích dữ liệu cho thấy, lãnh đạo là yếu tố tiếp theo ảnh hưởng đến chia sẻ tri thức với beta $=0.191$ và các giảng viên đánh giá với mức trung bình Mean =4,5872. Điều đó cho thấy, giảng viên chưa đánh giá cao vai trò của lãnh đạo trong hoạt động chia sẻ tri thức của mình. Nguyên nhân là do lãnh đạo không phải bao giờ cũng tôn trọng ý kiến, quan điểm của giảng viên, mức độ cởi mở của lãnh đạo với giảng viên cũng chưa cao. Lãnh đạo các khoa cần được trang bị các kỹ năng cần thiết như truyền thông, tạo động lực, lắng nghe để truyền cảm hứng cho các giảng viên tăng cường chia sẻ tri thức với nhau. Bản thân lãnh đạo phải là tấm gương chia sẻ tri thức. Nếu bản thân lãnh đạo không chia sẻ, luôn che dấu những thông tin, tri thức của mình thì không thể tạo ra văn hóa chia sẻ cho đơn vị mà mình phụ trách. Đồng thời lãnh đạo cần tạo điều kiện thuận lợi để các giảng viên có thêm nhiều cơ hội đào tạo, tập huấn cũng như tổ chức các hội nghị, hội thảo khoa học cho giảng viên để họ có cơ hội nâng cao năng lực chuyên môn đồng thời có cơ hội học tập chia sẻ tri thức với nhau, thúc đẩy hoạt động chia sẻ tri thức phát triển.

Thư tur, Nhà trường cần hiện đại hóa và nâng cao hiệu quả hoạt động của hệ thống thông tin. Kết quả phân tích dữ liệu cho thấy, hệ thống thông tin là yếu tố có mức độ ảnh hưởng thấp nhất đến chia sẻ tri thức với beta $=0,141$. Tuy nhiên, trên thực tế các giảng viên lại đánh giá yếu tố này ở mức trung bình cao so với các yếu tố khác Mean =5,0512. Điều đó chứng tỏ, các giảng viên xem hệ thống thông tin là một yếu tố hết sức quan trọng, là phương tiện để hoạt động chia sẻ tri thức diễn ra một cách thuận lợi và nhanh chóng. Mặc dù hệ thống thông tin đã được Nhà trường bắt đầu quan tâm xây dựng, tuy nhiên chỉ mới ở mức độ trung bình, vừa đủ. Do đó, ngoài việc trang bị những máy móc cần thiết, nhà trường cần lắp đặt thêm những thiết bị mới, nâng cao mức độ hiện đại hóa và hiệu quả hoạt động của hệ thống thông tin hỗ trợ cho nhu cầu công việc. Trường đại học cần đặc biệt chú ý phát triển hệ thống thông tin quản lý phục vụ công tác giảng dạy, nghiên cứu. Hệ thống bài giảng, học liệu cần được số hóa, cập nhật thường xuyên để giảng viên dễ dàng truy cập cũng như đóng góp tri thức của họ vào cơ sở dữ liệu dùng chung của tổ chức. Đặc biệt, hệ thống thông tin của trường đại học phải có tính tương tác cao, tạo điều kiện cho giảng viên có thể chia sẻ tri thức trực tuyến với nhau những thông tin, tri thức họ đang sở hữu.

\section{Hạn chế của đề tài:}

Mô hình gồm 04 yếu tố được kiểm định chỉ giải thích được 52,9\% sự biến thiên của việc chia sẻ tri thức. Chứng tỏ, ngoài 04 yếu tố này rất có thể còn có những yếu tố khác và các biến đo lường khác cũng tham gia vào việc giải thích về chia sẻ tri thức của giảng viên tại trường Đại học Tài chính - Marketing nhưng chưa được cô đọng trong mô hình nghiên cứu này.

Nghiên cứu chỉ được kiểm định tại trường Đại học Tài chính - Marketing đối với giảng viên, mà chưa được kiểm định cho các trường đại học khác, nên tính tổng quát hóa của kết quả nghiên cứu chưa cao. 


\section{Tài liệu tham khảo}

\section{Tiếng Việt}

Trần Minh Thành, 2013. Anh hưởng của các yếu tố văn hóa tổ chức đến chia sẻ tri thức-Nghiên cứu tại các doanh nghiệp xây dụng TP. Hồ Chí Minh, Luận văn Thạc sĩ, Đại học kinh tế Thành phố Hồ Chí Minh.

\section{Tiếng Anh}

Al-adaileh R, 2011. The impact of Organizational Culture on Knowledge Sharing: the Context of Jordan's Phosphate Mines Company. International Research Journal of Finance and Economics, Issue 63.

Al-Alawi, A. I., Al-Marzooqi, N. Y. \& Mohammed, Y.F., 2007. Organizational Culture and knowledge sharing: critical success factors. Journal of Knowledge Management, 11(2), pp. 22-42.

Alavi, M., Kayworth, T.R. and Leidner, D.E. 2006, "An empirical examination of the influence of organizational culture on knowledge management practices", Journal of Management Information Systems, Vol. 22, No. 3, pp. 191-224.

Becerra-Fernandez I., A. Gonzalez \& R. Sabherwal, 2004, Knowledge management: Challenges, Solutions and Technologies, Pearson Education Inc., pp. 10-25.

Bock, G.W., Zmud, R.W, Kim, Y.G., \& Lee, J.N., 2005. "Behavioral intention formation in knowledge sharing: Examining the roles of extrinsic motivators, social-psychological forces, and organizational climate", MIS Quaterly; 29(1), pp. 87-111.

Cabrera, E. F., \& Bonache, J., 1999. “An Expert HR System for Aligning Organizational Culture and Strategy", Human Resource Planning, 22 (1), 51-60.

Davenport, T.H. \& Prusak, L., 1998. Working knowledge: How organisation manage what they know. Harvard business school press, 23-76.

De Long, D. W. \& Fahey, L., 2000. Diagnosing cultural barriers to knowledge management, Academy of Management Executive, 14, 4, pp. 113-127.

Donate, M. J., \& Guadamillas, F., 2011. Organizational factors to support knowledge management and innovation, Journal of Knowledge management, 15(6), 890-914.

Eldridge, J.E.T. and Crombie, A., 1974. A Sociology of Organizations, Allen \& Unwin, London.

Greenberg, J. \& Baron, R. A., 2003. Behaviour in Organizations: Understanding and Managing the Human Side of Work. New Jersey: Pearson Education Inc.

Gupta, A. K., and Govindarajan, V., 2000. “Knowledge management's social dimension: Lesson from Nucor Steel”, Sloan Management Review, 42 (1) 71-80.

Islam, Z. M., Hasan, I., Ahmed, S. U., \& Ahmed, S. M., 2011. Organizational culture and knowledge sharing: Empirical evidence from service organizations. African Journal of Business Management, 5(14), 5900-5909. 